Reprinted (adapted) with permission from

ACS Nano, 2016, 10 (3), pp 3365-3374

DOI: $10.1021 /$ acsnano.5b07162

Publication Date (Web): February 22, 2016

Copyright (C 2016 American Chemical Society

\title{
Effect of Actin Organization on the Stiffness of Living Breast Cancer Cells Revealed by Peak-Force Modulation Atomic Force Microscopy
}

\author{
Alicia Calzado-Martínt, Mario Encinarł, \\ Javier Tamayo, Montserrat Calleja, Alvaro San Paulo*
}

Instituto de Microelectronica de Madrid (IMM,CSIC),

Isaac Newton 8, 28760, Tres Cantos, Madrid. Spain

*Corresponding Author's E-mail address: alvaro.sanpaulo@csic.es 


\section{Keywords:}

Atomic Force Microscopy, Peak-Force, Live Cell Imaging, Cell Mechanics, Cancer Mechanics 


\begin{abstract}
We study the correlation between cytoskeleton organization and stiffness of three epithelial breast cancer cells lines with different degree of malignancy: MCF-10A (healthy), MCF-7 (tumorigenic/non-invasive) and MDA-MB231 (tumorigenic/invasive). Peak-force modulation atomic force microscopy is used for high-resolution topography and stiffness imaging of actin filaments within living cells. In healthy cells, local stiffness is maximum where filamentous actin is organized as well-aligned stress fibers, resulting in apparent Young's modulus values up to one order of magnitude larger than in regions where these structures are not observed. But these organized actin fibers are barely observed in tumorigenic cells. We further investigate cytoskeleton conformation in the three cell lines by immunofluorescence confocal microscopy. The combination of both techniques determines that actin stress fibers are present at apical regions of healthy cells, while in tumorigenic cells they appear only at basal regions, where they cannot contribute to stiffness as probed by atomic force microscopy. These results substantiate that actin stress fibers provide a dominant contribution to stiffness in healthy cells, while the elasticity of tumorigenic cells appears not predominantly determined by these structures. We also discuss the effects of the high-frequency indentations inherent to peak-force atomic force microscopy for the identification of mechanical cancer biomarkers. Whereas conventional low loading rate indentations $(1 \mathrm{~Hz})$ result in slightly differentiated average stiffness for each cell line, in high-frequency measurements $(250 \mathrm{~Hz})$ healthy cells are clearly discernible from both tumorigenic cells with an enhanced stiffness ratio; however, the two cancerous cell lines result undistinguishable.
\end{abstract}


The treatment of the living cell as a biophysical system is a very fertile paradigm in understanding cell biology. In particular, many diseases have been found to be related with alterations of the mechanical properties of cells. ${ }^{1-5}$ Cancer is a paradigmatic case in this respect: tumor cells with different degree of malignancy exhibit different stiffness, different states of cytoskeletal tension and many other mechanical alterations. In consequence, the comparative analysis of cell mechanics in normal vs. diseased cells represents a meaningful approach towards both understanding the physical nature of cancer and exploring novel diagnosis methods. ${ }^{6-9}$ A wide variety of experimental techniques have been developed in order to assess cell mechanics at different length and time scales. ${ }^{10-13}$ Among these, atomic force microscopy (AFM) outstands for two particular capabilities: high resolution imaging and quantitative characterization of cell mechanics. ${ }^{14,15}$ Cell stiffness, the resistance of the cell to an externally induced deformation, is measured in AFM experiments by monitoring the force exerted on a probe cantilever as its distance to the cell is reduced and a tip placed at its end produces an indentation. A larger resistance to deformation results in a larger force, measured as a larger deflection of the cantilever. One of the earliest applications of AFM for quantitative cell mechanics was indeed a comparative study of cancer cells with different degree of malignancy. ${ }^{16}$ It was clear since these early experiments that differences between normal and cancerous cells were evident in force-distance (FD) curves: the slope of the curves acquired on cancerous cells, and hence their resistance to deformation, was systematically smaller than that corresponding to normal cells. Experimental FD curves acquired during cell indentations can be very accurately fitted to equations derived from theoretical models that describe their elastic response in terms of an apparent Young's modulus, $E$. For several years, progressive advances have been made to improve the accuracy and consistency of measurements and modeling of apparent Young's modulus on living cells. ${ }^{17}$ These include considering the effect of tip geometry, ${ }^{18}$ indentation depth, ${ }^{19}$ loading rate, ${ }^{20}$ prolonged poking, ${ }^{17}$ substrate stiffness ${ }^{21}$ and others. ${ }^{22}$ In parallel, many works have been devoted to characterize the differences in apparent Young's modulus between normal and cancerous cells of different types, including actual samples from cancer patients. ${ }^{17,23-29}$ These works have revealed a consistently lower stiffness of cancer cells as compared to healthy cells regardless of the cancer type, which has motivated an on-going research about the suitability of cancer biomarkers based on mechanical cell properties. ${ }^{30,31}$ However, the underlying causes for this reduced stiffness of cancer cells versus their normal counterparts, as well as its cause-effect relationship with other conformational changes observed during cancer growth and metastasis, remain not fully elucidated. Human breast epithelial cells 
represent an exemplary case regarding this issue. Is has been proposed that the lower stiffness of breast cancer cells is related to a reduction in the presence of well-organized filamentous actin (F-actin) stress fibers, a key constituent of the cytoskeleton system which greatly determines mechanical cell elasticity. ${ }^{32}$ The cytoskeleton is a dynamic structure that continuously reorganizes its architecture into specific functional arrangements. Actin is organized in two main structures, bundles or networks, which play different roles within cells. Stress fibers are long contractile actin bundles found in non-muscle cells cross-linked into closely packed parallel arrays. On the other hand, the cell cortex under the membrane is mainly composed of networks of loosely cross-linked F-actin, with similar physical properties to those of semisolid gels. The reduction of the presence of actin stress fibers observed in breast cancer cells is expected to result in a weaker cytoskeletal structure, thus providing malignant transformed adherent cells with a high potency to migrate similar to that of motile cells. ${ }^{20}$ However, a direct correlation between the differences in F-actin organization in living healthy and tumor cells and their differences in stiffness has not been observed yet.

Correlating cell stiffness with cytoskeletal conformations in living cells requires the ability to obtain high resolution apparent Young's modulus images of cell regions where specific cytoskeletal structures can be identified. In fact, high resolution imaging of cytoskeletal constituents with stiffness contrast of living cells can be expected to provide very meaningful comparisons between different regions of the cells, thus helping to understand the origin of the differences in stiffness among normal and cancer cells, and opening new ways to correlate differences in mechanical properties with other dissimilarities. But this kind of experiments have remained elusive for cell characterization methods so far. Apparent Young's modulus maps of cells can be obtained by so-called FD curve-based AFM imaging methods. ${ }^{33,34}$ These methods are based on the acquisition of an array of FD curves over a cell. Each of these curves contains information about the local cell stiffness at each image pixel. Until only a few years ago, the most extended FD imaging method, usually referred to as force-volume mode, ${ }^{24,25}$ consisted on the acquisition of a small array of low loading rate FD curves (typically $1 \mathrm{~Hz}$ ). This implied very poor resolution (normally a few tens of pixels per scan line for a scan size of tens of micrometers, resulting in a lateral resolution of a few micrometers) and long image acquisition times (several tens of minutes). Such a low resolution was a limiting issue in order to identify cytoskeletal structures and to measure the corresponding local stiffness. A significant breakthrough for the acquisition of high 
resolution stiffness images at high speed has been recently accomplished with the merge of FD curve-based imaging with dynamic modes. Dynamic AFM modes are based on AC actuation on the cantilever, so that any distance-dependent characteristic magnitude of the resulting motion can be employed as feedback parameter. The most extended dynamic AFM modes, amplitude modulation (AM) and frequency modulation (FM), rely respectively on controlling either the amplitude or resonance frequency of the probe, which oscillates at or very close to its resonance frequency. ${ }^{35}$ But direct apparent Young's modulus imaging from real-time acquired FD curves results impractical in AM and FM modes, although recent works have made important progress to develop AM mode based methods that can provide high resolution stiffness imaging by other approaches. ${ }^{36-39}$ A more recently developed dynamic AFM mode provides a straightforward approach to high resolution and fast acquisition FD curve-based imaging. As it is shown in Figure 1, this mode uses the maximum repulsive force registered during each actuation cycle as the feedback parameter, so that it can be referred to as peak-force modulation (PFM) mode. ${ }^{40}$ In PFM-AFM, the probe is actuated well below resonance, at frequencies in the 0.1 to $1 \mathrm{kHz}$ range, and the AFM controller registers the cantilever deflection along the whole actuation cycle. A topography image is built from the voltage applied to the $\mathrm{Z}$ piezo scanner so that the peak value of the force for each actuation cycle is kept constant along XY scanning. High resolution apparent Young's modulus imaging is straightforward in PFM-AFM because the instrument registers a large array of complete FD curves, one for each image pixel, and a real-time fitting to any theoretical equation of choice is performed. The resulting stiffness map is displayed simultaneously to the topography image. The high actuation frequency used for curve acquisition in PFM-AFM enables high resolution imaging (up to thousands of pixels per line for a scan size of tens of micrometers, resulting in a lateral resolution of tens of nanometers) combined with fast acquisition times (a few minutes per image). Apparent Young's modulus imaging based on PFM-AFM is currently implemented as a standard feature in several commercially available instruments under different denominations. This AFM operation mode offers an unprecedented potential for high-resolution, label-free, topography and stiffness contrast imaging of cytoskeletal structures within living cells. However, optimizing image acquisition and quantitative measurements on living cells in liquid media is not a trivial issue, and only a few works have been reported so far. ${ }^{41-49}$ 


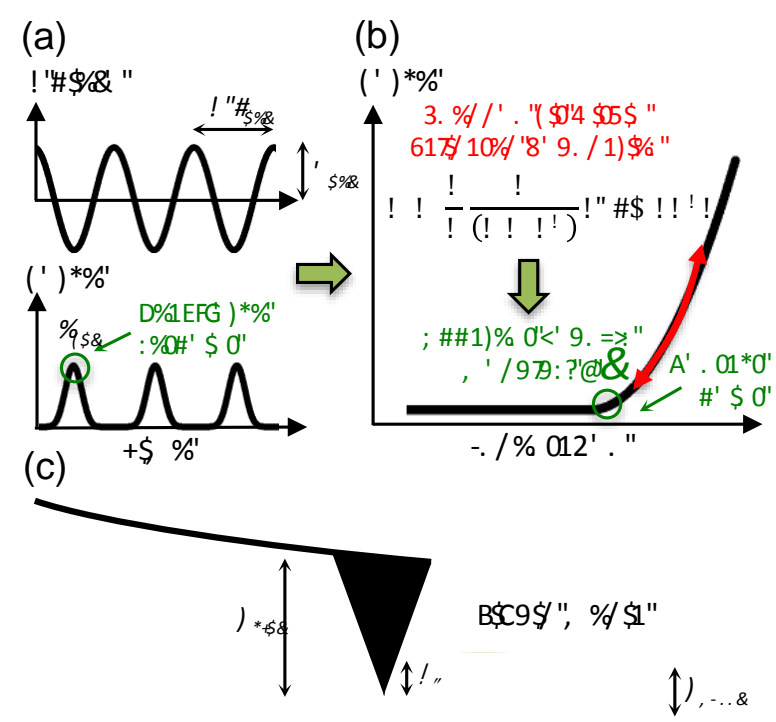

398: 0) 10\%'

Figure 1. Schematic representation of the operation of peak-force modulation AFM for imaging living cells in liquid media. (a) The peak values of the periodic force $\left(F_{S P}\right)$ resulting on the tip as a consequence of sinusoidal actuation on the cantilever (at frequency $f_{P F}$ with amplitude $A_{P F}$ ) are used for feedback control. (b) Real-time measurements of the force vs. the tip-sample distance are converted into force-indentation curves that are fitted to the Sneddon equation for a conical indenter, so that the resulting values of the apparent Young's modulus $E$ are displayed as an image. (c) The probe and the operating conditions are chosen so that the tip height $\left(h_{T I P}\right)$ and the amplitude $\left(A_{P F}\right)$ are larger that the maximum cell height $\left(h_{C E L L}\right)$.

In this work we show the application of PFM-AFM for imaging cytoskeleton structures within living breast cancer cells corresponding to cell lines with different degree of malignancy: MCF-10A (healthy), MCF-7 (tumorigenic, non-invasive) and MDA-MB-231 (tumorigenic, invasive). In particular, we show the capability of PFM-AFM for highresolution mapping of the local stiffness of cell regions where organized F-actin structures are identified, and then compare the results with areas where these structures are not observed. Furthermore, we compare the presence of F-actin structures in PFM-AFM images from all three cell lines, and correlate the results with the complementary characterization of the cytoskeleton arrangement by immunofluorescence confocal microscopy. We observe significant differences between healthy and tumor cells in the conformation of the F-actin cytoskeleton structures, which contribution to cell stiffness is discussed in consideration of the PFM-AFM apparent Young's modulus imaging results. Finally, we discuss the effects on 
the observed cell stiffness of the high frequency probe actuation characteristic of PFM-AFM, which we find to have an impact on both absolute values and ratios among the apparent Young's modulus of the three cell lines. This has implications on the use of local stiffness measurements by high frequency indentations for the identification of cancer biomarkers based on the mechanical elasticity of cells.

\section{RESULTS AND DISCUSSION}

\section{Measurement of Apparent Young's Modulus}

Figure 2 shows an example of the FD curves obtained while imaging MCF-10A cells. The software used includes a function that removes in real time the hysteresis effect caused by hydrodynamic damping with the liquid media (see methods). As a result, approach and retract curves typically overlap, as the example in fig. 2(a). Tip-surface indentation is calculated as the difference between the z-piezo position and the cantilever deflection. We find that the slope of the resulting force-indentation curves is best fitted to a quadratic behavior, which justifies the use of the Sneddon equation (conical indenter) for extracting values of the apparent Young's modulus:

$$
F=\frac{2}{\pi} \frac{E}{\left(1-v^{2}\right)} \tan \alpha \cdot \delta^{2}
$$

where $F$ is the force, $E$ is the apparent Young's modulus, $v$ is the Poisson's ratio (we assume $v=0.5$ for all cases), $\alpha$ is the tip half angle and $\delta$ is the indentation. The fact that this equation provides good fitting to the experimental curves is itself a remarkable result, because the actuation frequency used in PFM-AFM is more than 100 times larger than the loading rate used in typical FD curve-based indentation experiments. Is such conditions, a large contribution of viscoelastic tip-cell interaction can be expected, and this might influence the

functional behavior of force $v s$. indentation. ${ }^{50}$ Regardless of the cell line, we find that the high actuation frequency used does not perturb the Sneddon-like quadratic behavior of the curves, which motivates to continue using the apparent Young's modulus as given by the Sneddon equation as a measure of the resistance to deformation of the cells in PFM-AFM experiments. 

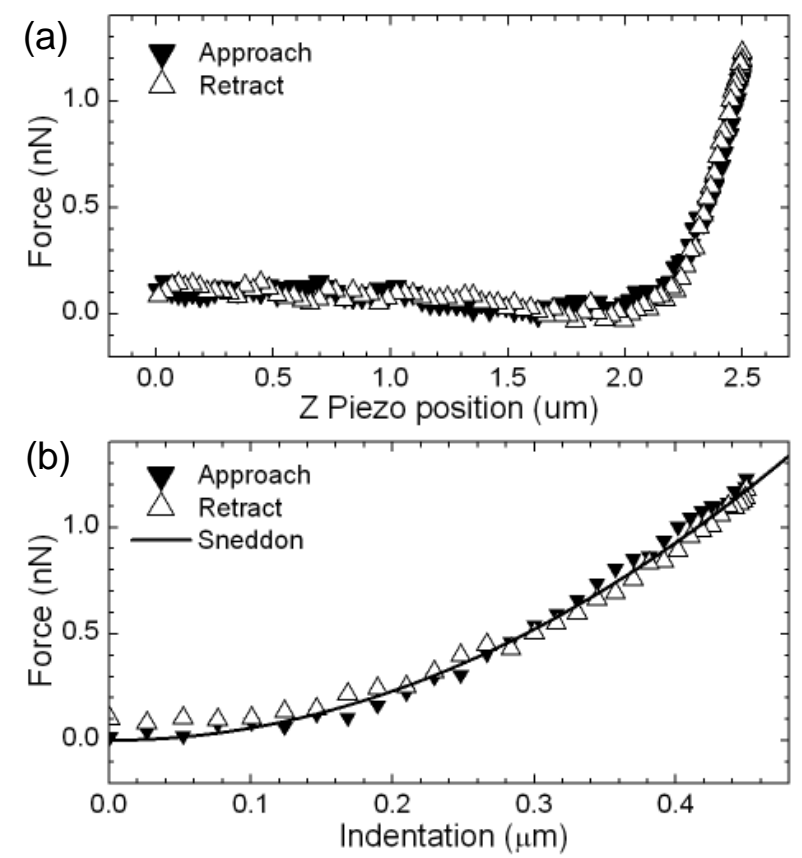

Figure 2. Representative example of experimental curves obtained on living cells by PFM-AFM. (a) Force $v s$. z-piezo position curves on a MCF-10A cell. (b) Resulting force-indentation curves showing a quadratic behavior consistent with a conical indentation as given by the Sneddon equation.

A key issue for generating consistent images of apparent Young's modulus is considering the effect of indentation depth. We have addressed this point by checking the resulting values for $E$ at different indentation depths. A valid range is established by determining the values for which $E$ is independent of the indentation depth. This procedure has been successfully applied in previously reported works for the same MCF-10A and MCF-7 cell lines considered here. ${ }^{51}$ An example for a MCF-10A cell is shown in Figure 3, where average values for $E$ are represented versus the average relative cell deformation produced. The relative cell deformation is defined as the percentage of indentation depth relative to the cell height as given by the topography data. Figure 3 shows that similar results are obtained for lower peripheral membrane regions and for higher apical areas of the cells. For small indentations (resulting in relative deformations below 5-7\%), an irregular tip-sample contact with a not well-defined geometry can be expected. As a result, the obtained values for $E$ are not constant and show a region of monotonic increase. As the indentation increases, the plots show a plateau where $E$ is approximately constant for indentations in the range from $7 \%$ to $12 \%$. For even larger indentations, a noticeable increase of $E$ in observed in both areas for the three cell lines. Several effects might contribute to this increase, ${ }^{22}$ but a dominant effect of 


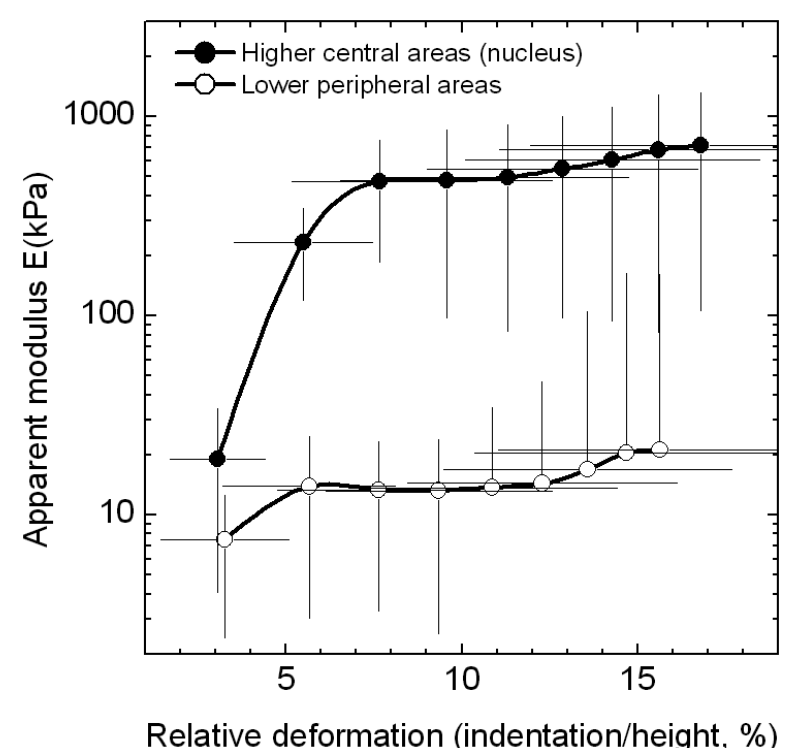

Figure 3. Observed behavior of the measured apparent Young's modulus $E$ as a function of the indentation depth, normalized to the cell height. Regardless of the region of the cell, and for all the three cell lines considered, we find a plateau region where $\mathrm{E}$ is approximately independent of the indentation. In all cases, a relative deformation of around $10 \%$ ensures a consistent determination of $E$.

substrate induced stiffening is always expected. ${ }^{21}$ The plateau region where $E$ is independent of indentation sets a valid range for obtaining meaningful images and for a consistent comparison of stiffness among the cell lines. In general, we find that a relative deformation of around $10 \%$ ensures a consistent estimation of $E$ regardless of the cell line. In our experiments, this value corresponds to an indentation force of $0.3-0.5 \mathrm{nN}$. Since the peakforce setpoint is set at $0.7-1 \mathrm{nN}$, the determination of $E$ for such reduced values of the force is made by setting an upper force fitting boundary of around $50 \%$ of the setpoint in the forceindentation curves. The lower limit is set at $10 \%$ to prevent the effect of sporadic irregular behaviors at the contact point.

\section{Comparative Analysis of PFM Mode AFM Images from Different Cell-Lines}

The panels of figures 4 and 5 present a comparison by columns of representative images from each of the cell lines considered in this work (further images are provided in figures S3, S4 and S5 of the supplementary information). A total of 69 image acquisitions have been analyzed in this study. The images correspond to topography (first row) and apparent Young's modulus (second row). The topography image of healthy MCF-10A cells in fig. 4(a) 
shows two adjacent cells with lobular shape, around 20 micrometers wide and 30 micrometers long, with a maximum height at the central nuclear region of around 6 micrometers. The most prominent feature observed in MCF-10A images is the appearance of two different kinds of filamentous structures. In some areas, such as the left side region of the lower left cell in fig. 4(a), the presence of long, compact and well-aligned fibers is observed. In other areas, for instance at the central higher region of the same cell, shorter, more dispersed and disorganized network structures are found. In general, we do not observe any correlation between the presence of each type of structure and the height of the cell. Both types of arrangements, fibers and networks, were observed in higher perinuclear and lower membrane regions of the cells. Figure 5(a) displays a zoomed region of the topography image corresponding to fig.4(a) where the two kinds of structures appear close to each other. This image has been high-pass filtered in order to enhance the contrast produced by these filamentous structures. The high resolution of the image allows to determine an apparent width of the filaments arranged as fibers in the range of $300-500 \mathrm{~nm}$, and a height over the cell surface of around $25-50 \mathrm{~nm}$. These structures resemble the expected morphology for actin stress fibers, as previously imaged by various AFM modes. ${ }^{20,49,52}$

An apparent Young's modulus image simultaneously acquired to the topography is shown in Figure 4(d). Remarkably, the contrast observed in the apparent Young's modulus image is correlated to the features observed in the topography image. The areas with filaments arranged as fibers systematically correspond to larger values of $E$ (clearer tones). Actually, the high resolution of the apparent Young's modulus image allows identifying the morphology of these fibers also in the elasticity image, as clearly seen in the zoomed-in area of Figure 5(d). On the contrary, the disorganized network areas observed in topography are correlated with lower values of $E$ in the elasticity image (darker tones), but the contrast pattern observed in topography images is not reproduced in the elasticity images at this regions. The section profiles in figures $4(\mathrm{~g})$ and $4(\mathrm{e})$ illustrate how the stiffer regions correspond to either lower or higher parts of the cells. The apparent Young's modulus measured in the areas with well-aligned fibers is in the range of $80-200 \mathrm{kPa}$, whereas it drops around one order of magnitude to $10-30 \mathrm{kPa}$ in the areas with disorganized networks. This result was reproduced in all images obtained in MCF-10A cells. Systematically, cell stiffness was found to be around 7-10 times harder in areas where well-aligned fibers were observed than in areas where disorganized networks were present. 


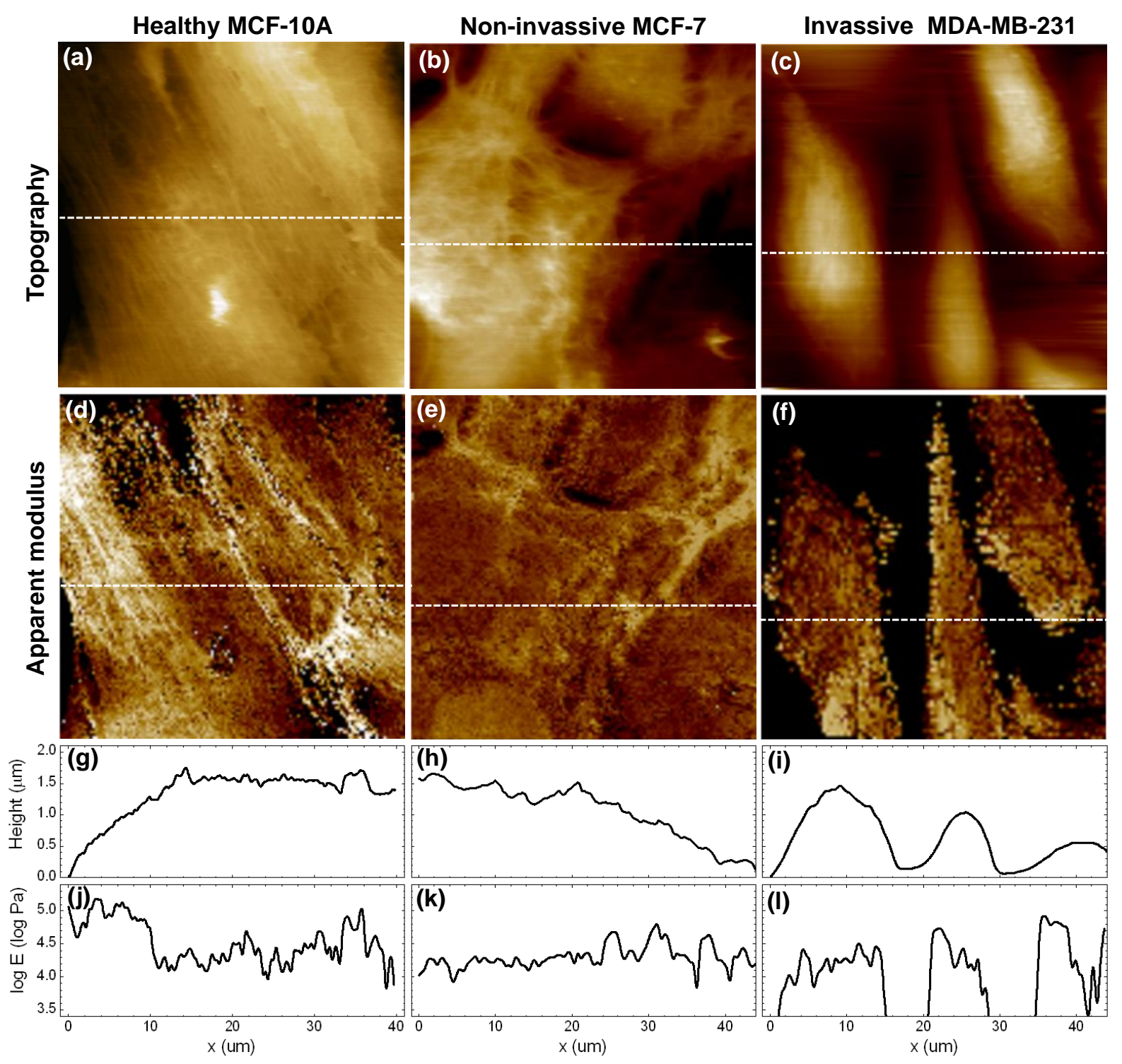

Figure 4. Comparative Imaging results for the three cell lines considered. First column images $(a, d)$ and cross-section profiles (g,j) correspond to MCF-10A cells. Second column images (b,e) and crosssection profiles (h,k) correspond to MCF-7 cells. Third column images (c,f) and cross-section profiles (i,l) correspond to MDA-MB-231cells. First row images (a,b,c) show topography contrast, while second row images $(\mathrm{d}, \mathrm{e}, \mathrm{f})$ show apparent Young's modulus contrast. Image size is $40 \mathrm{x} 40 \mu \mathrm{m}$ for MCF-10A and $45 \times 45 \mu \mathrm{m}$ in all other cases. Topography range is $2 \mu \mathrm{m}$ for MCF-10A and $6 \mu \mathrm{m}$ in all other cases. Apparent Young's modulus images are displayed in a logarithmic color scale, from 2.5 to $250 \mathrm{kPa}$. Data corresponding to the substrate in (f) are removed for clarity. 

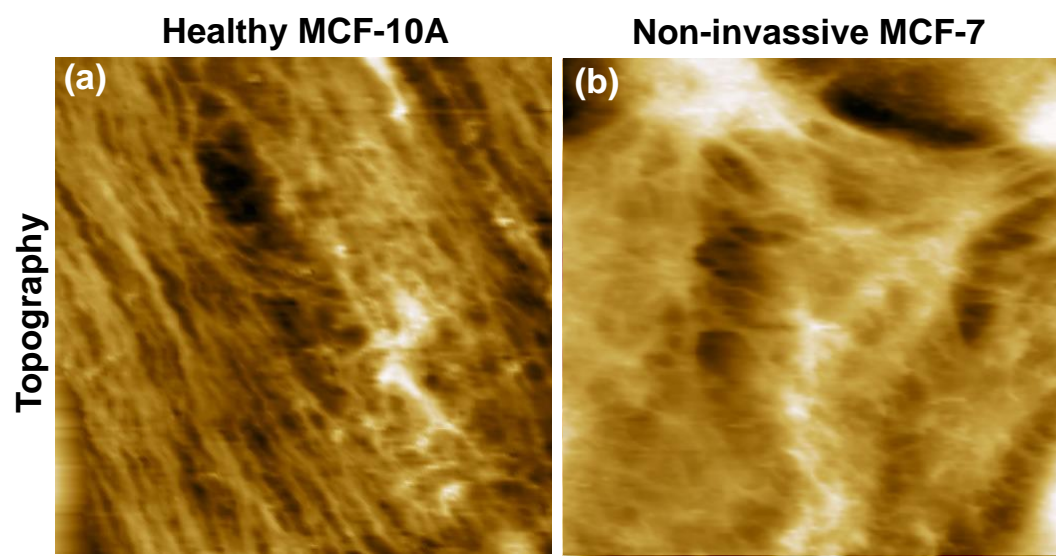

Invassive MDA-MB-231
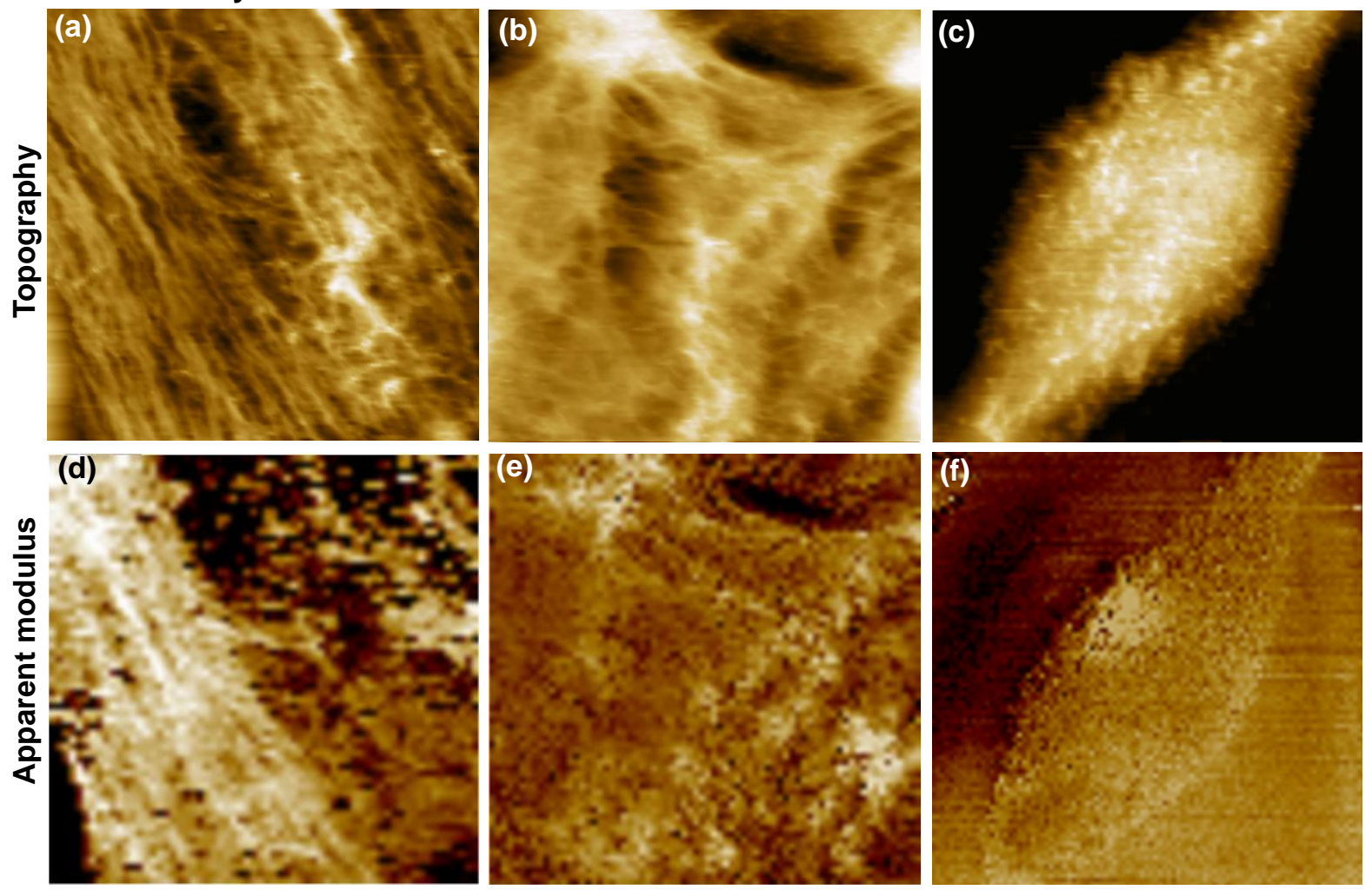

Figure 5. High magnification images showing examples of the distinctive contrast patterns observed for each cell line. First column images (a,d) correspond to MCF-10A cells. Second column images (b,e) correspond to MCF-7 cells. Third column images (c,f) correspond to MDA-MB-231 cells. First row images $(a, b, c)$ show topography contrast, while second row images $(\mathrm{d}, \mathrm{e}, \mathrm{f})$ show apparent Young's modulus contrast. Image size is $25 \times 25 \mu \mathrm{m}$ in all cases. Topography range is $2 \mathrm{um}$ for MCF-10A, $4 \mathrm{um}$ for MCF-7 and 6 um for MDA-MB-231. Apparent Young's modulus images are displayed in a logarithmic color scale, from 2.5 to $250 \mathrm{kPa}$.

Filamentous structures arranged as well-aligned fibers were almost exclusively observed for the healthy MCF-10A cell line, and barely found on any of the two tumorigenic cell-lines. In fact, the topography and apparent Young's modulus images of both MCF-7 and MDAMB-231 cell lines present hardly distinctive patterns, as shown in the examples displayed in figures 4(b), 4(c), 4(e) and 4(f). The overall dimensions and height in both cases are similar to those of healthy cells, but tumorigenic cells show a much featureless morphology. Only in some cases, as shown in fig 5(b), some areas of MCF-7 cells present disorganized network structures somehow similar to those found on MCF-10A cells. Similarly, some MDA-MB231 cells show a granular texture along its surface, as in the example shown in fig 5(c), very different from the well-aligned filaments observed in healthy cells. However, for both 
tumorigenic cell lines there is hardly any significant correlation between these topographic features and the contrast observed in the apparent Young's modulus images. For MCF-7 and MDA-MB-231 cells, the only systematic observation is that the few observed stiffer regions with fiber-like morphology always correspond to lower heights of the cells. For the central, higher nuclear regions of MCF-7 and MDA-MB-231 cells we obtain values of $E$ in the range of 10-30 kPa, matching the softer areas observed in MCF-10A cells. For the few stiffer regions observed in some of the outer and lower areas of MCF-7 and MDA-MB-231 cells, the values of $E$ reach a range of $30-100 \mathrm{kPa}$.

\section{Correlation of PFM-AFM Images with Immunofluorescence Assays}

The images obtained by PFM-AFM point out to differences in the arrangement of filamentous actin between healthy and tumor cells as responsible for the measured differences in apparent Young's modulus. ${ }^{20}$ We have confirmed this hypothesis by characterizing the cytoskeletal organization within MCF-10A, MCF-7 and MDA-MB-231 cells in vitro by immunofluorescence assays. The conformations of F-actin and acetylated $\alpha$ tubulin, a specific marker for stable microtubules (MTs), were evaluated by confocal microscopy, and representative results are shown in figure 6 (additional images are provided in figure S4 of the supplementary information). Regardless of the cell line, MTs (green stained) are observed as fibrous networks arising from the perinuclear region and spreading randomly to the cytoplasm of the cell, and no systematic differences are detected among the three cell lines. On the contrary, important differences are found in the arrangement of Factin (red stained) between the healthy and the two tumor cell lines. In the case of MCF-10A cells, F-actin is either organized in parallel stress fibers or as short and disperse fibers. Remarkably, actin stress fibers are distributed throughout the cell body, and a number of MCF-10A cells exhibit long stress fibers even in the higher nuclear regions. While healthy cells organize most of their actin in well-defined fibers, both cancer cell lines exhibit less prominent F-actin structures with a diffuse distribution in their cytoplasm, which we attribute to a weaker localization in the cortical cytoskeleton. Moreover, the scarce long actin bundles found in tumor cells are mainly confined to cell borders or ventral regions, but no long stress fibers were detected in cortical regions. 

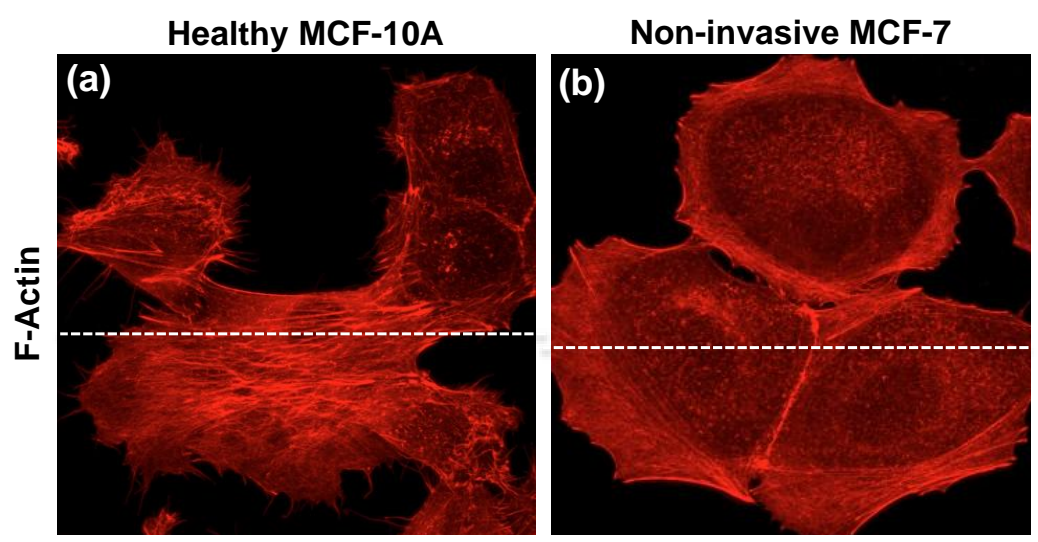

Invasive MDA-MB-231
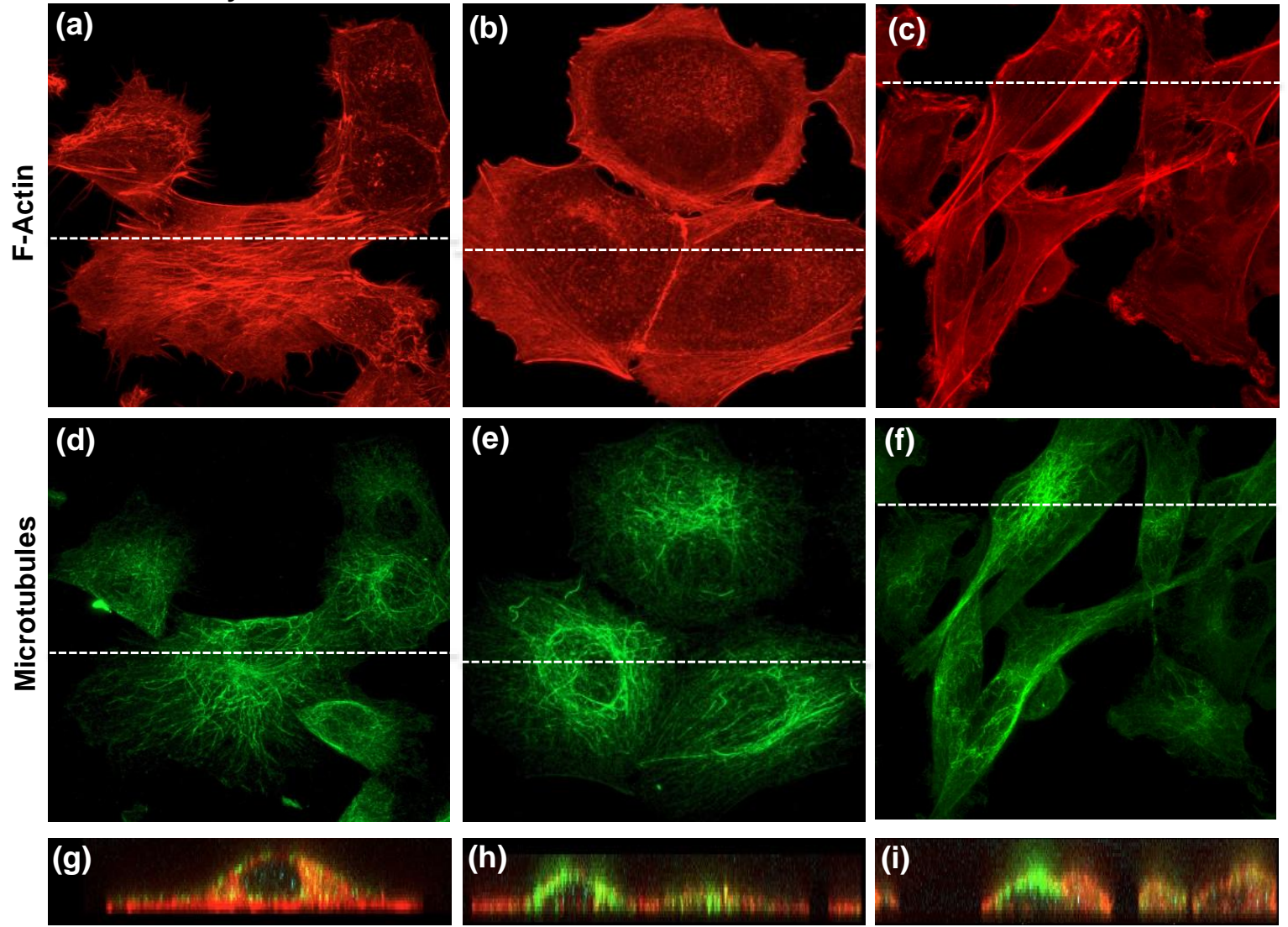

Figure 6. Confocal fluorescence microscopy images of cells from the three lines considered. First column images $(\mathrm{a}, \mathrm{d})$ and orthogonal projections (g) correspond to MCF-10A cells. Second column images (b,e) and orthogonal projections (h) correspond to MCF-7 cells. Third column images (c,f) and orthogonal projections (i) correspond to MDA-MB-231cells. First row images (a,b,c) show red-stained F-actin filaments, while second row images $(\mathrm{d}, \mathrm{e}, \mathrm{f})$ show green-stained microtubules. Image size is $80 \times 80$ um in all cases. Both contrast signals are mixed in the orthogonal projections.

A three-dimensional understanding of the distribution of both components within the cells can be accomplished by looking at the reconstructed orthogonal projections displayed in Fig. 6 (g,h,i). According to these projections, it becomes clear that the surface of MCF-10A shows higher content of F-actin than tumor cells. Also, the projections for MCF-10A reveal that Factin organized as stress fibers is found in basal regions closer to the substrate, but it is also noticeably present in apical regions alongside the higher perinuclear areas. On the contrary, both tumorigenic cell lines show a limited presence of actin stress fibers in the perinuclear apical regions. Instead, disperse actin networks and MTs dominate the cytoskeleton arrangement in these areas, and actin stress fibers appear almost exclusively at the lower basal regions. 
The complementary information provided by PFM-AFM and confocal microscopy allows identifying unequivocally the long, compact and well-aligned filamentous structures observed by PFM-AFM in the healthy cells as the red-stained F-actin stress fibers visualized in the immunofluorescence assays. In consequence, the different contrast patterns observed in the PFM-AFM elasticity images and their correlation to topography are attributed to differences in the arrangement of F-actin stress fibers between healthy and tumor cells. In MCF-10A cells, F-actin stress fibers produce measurements of apparent Young's modulus that are up to one order of magnitude higher that those in regions where these structures are not observed. But these well-aligned F-actin fibers are observed at apical regions only in healthy cells, and they are not found in such regions in any of the tumorigenic cell lines. F-actin stress fibers remain at basal regions in both tumorigenic cell lines, where they cannot contribute to cell stiffness as measured by any AFM-based indentation experiment. These results determine that stress fibers provide a dominant contribution to cell stiffness in healthy MCF-10A cells, while MCF7 and MDA-MBB-231 elasticity seems not primarily influenced by these components. In consequence, the configuration of F-actin filaments is proved as an essential factor for the higher stiffness of normal vs. cancer cells systematically reported in previous AFM studies. Another consequence of these results concerns the ability to image cytoskeleton structures within living cells. To date, most of the work done on cytoskeleton mechanics consists in the evaluation of its components individually or in artificial networks. ${ }^{53,54}$ It is known that actin filaments are much less rigid than MTs. ${ }^{55,56}$ However, the mechanical properties of cytoskeletal structures within living cells strongly rely on other important factors such as motor proteins, crosslinking or hydrostatic pressure. ${ }^{57}$ These elements promote the assembly of highly organized and stiff F-actin structures. Our results demonstrate that PFM-AFM is capable of evaluating the contribution of these organized structures to cell stiffness within whole living cells in fully native conditions, where the effect of all these factors is naturally into play. 


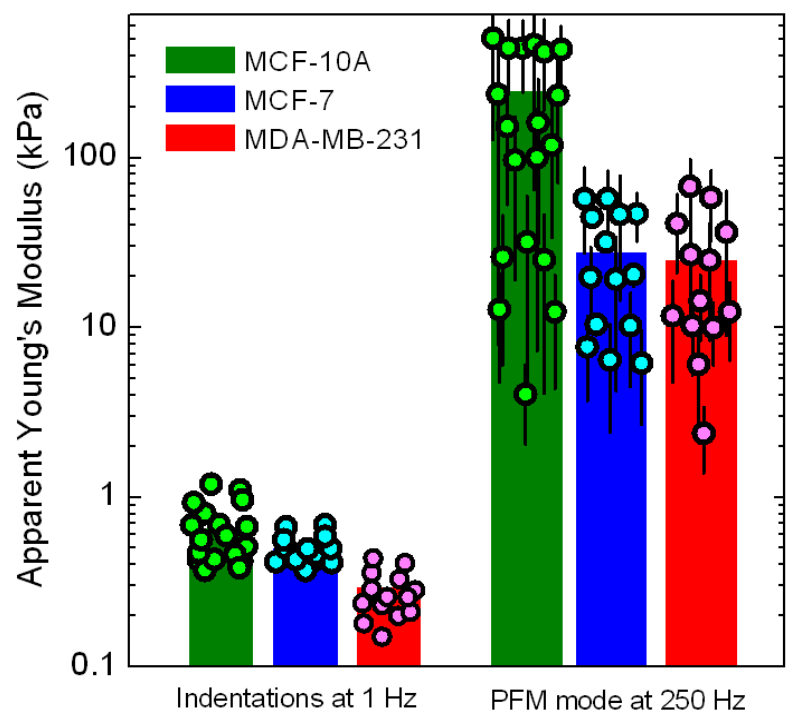

Figure 7. Comparison of average values of apparent Young's modulus obtained for single cells by either low loading rate indentations at $1 \mathrm{~Hz}$ or peak-force modulation mode at $250 \mathrm{~Hz}$. Each point represents the average apparent Young's modulus of a single cell.

\section{Comparing PFM-AFM with low loading rate indentation experiments}

One remarkable observation from the presented result is the difference in the values obtained in all cases for $E$ as compared to typical values previously reported for these cell lines. Reported values are in the order of $1 \mathrm{kPa}$ as obtained by conventional low loading rate indentations. ${ }^{13,20}$ We have confirmed this discrepancy by performing a series of low loading rate indentation experiments on each cell line with the same cell culturing conditions as used in the PFM-AFM imaging experiments. The resulting values for $E$ are displayed in figure 7. In order to compare the results obtained by each method, averaged values of $E$ as obtained by PFM-AFM are also shown. Note that in the case of the low loading rate indentations, each point represents the average $E$ obtained from a few force-indentation curves performed on a single cell with a large radius tip (see methods). In those conditions, each indentation probes the mechanical response of the cell as a whole. In the case of PFM-AFM results, each point corresponds to the average $E$ obtained from the set of curves that comprises a PFM-AFM image of a whole single cell, typically around $10^{4}-10^{5}$ curves obtained with a sharp tip. In this case, each indentation probes the local stiffness at each point of the cell. In addition, the tip follows a triangular wave form in the low loading rate experiments, resulting in a constant 
loading rate of $1 \mathrm{~Hz}$, whereas PFM-AFM indentations follow a sinusoidal motion which implies a non-constant loading rate. Regardless of these disparities in the experimental procedures and in the interpretation of $E$ for each case, it is interesting to discuss the differences found.

There are two prominent differences between the results obtained at $1 \mathrm{~Hz}$ indentations and the $250 \mathrm{~Hz}$ PFM-AFM experiments. The first one is the stiffening effect for all cell lines, and the second one is the change in the ratios of stiffness between them. In the case of low loading-rate indentation, healthy $\mathrm{MCF}-10 \mathrm{~A}$ cells present an average modulus of $0.7 \pm 0.3$ $\mathrm{kPa}$, whereas cancerous specimens display narrower distributions centered at slightly lower values, with results of $0.5 \pm 0.1 \mathrm{kPa}$ for MCF-7 cells and $0.3 \pm 0.1 \mathrm{kPa}$ for MDA-MB-231 cells. Notably, cell stiffness is found to scale down with the degree of malignancy: healthy MCF-10A cells are stiffer than any of the cancerous cells, while non-invasive MCF-7 cells are also stiffer than invasive MDA-MB-231 cells. This picture changes significantly with measurements obtained by PFM-AFM. For those, healthy MCF-10A cells are significantly stiffer than any of the tumor cells, with an average value of $250 \pm 100 \mathrm{kPa}$. However, both cancerous cell lines show similar average elastic modulus values, with values of $28 \pm 12 \mathrm{kPa}$ and $25 \pm 13 \mathrm{kPa}$ for MCF-7 and MDA-MB-231 respectively. In consequence, our results reveal an apparent stiffening effect for all cell lines when probed by PFM-AFM at $250 \mathrm{~Hz}$ as compared to the indentations at $1 \mathrm{~Hz}$. Stiffening due to increasing loading rate is actually an expected effect for cells due to their viscoelastic response. A stiffening effect in MCF-10A and MCF-7 cells produced by an increasing loading rate in the range from 0.03 to $1 \mathrm{~Hz}$ had already be reported..$^{20}$ The apparent Young's modulus of MCF-10A and MCF-7 cells was found to increase by a factor of around 2.1 from $0.03 \mathrm{~Hz}$ to $1 \mathrm{~Hz}$ for both lines. In AFMbased microrheology experiments made on the same three cell lines considered in this work, it was found that both the storage and loss modulus (respectively a measure of the elastic and viscous response of the cell to small harmonic deformations) increased more than one order of magnitude for deformations made from 1 to $100 \mathrm{~Hz} .^{58}$ In our case, we estimate an increase by a factor of around 350 for the healthy cells and by a factor of 60 for the tumorigenic cells when comparing the indentations at $1 \mathrm{~Hz}$ with the PFM mode experiments at $250 \mathrm{~Hz}$. Another consequence of probing cell stiffness at higher frequency is that it increases the ratio of the apparent Young's modulus between normal and tumorigenic cells. According to our results, the ratio of the average stiffness between MCF-10A and MCF-7 increases from 1.4 at $1 \mathrm{~Hz}$ to 8.9 at $250 \mathrm{~Hz}$. In consequence, if AFM measured cell stiffness was used as a cancer 
biomarker, it should be considered that the difference between healthy and tumor cells is amplified at increasing loading rates. However, the opposite happens when considering the differences in stiffness between non-invasive MCF-7 cells and invasive MDA-MB-231 cells: according to our results, while the ratio between their apparent Young's modulus at $1 \mathrm{~Hz}$ is 1.6, its value drops to 1.2 at $250 \mathrm{~Hz}$.

\section{CONCLUSION}

The conclusions of this work can be summarized in three points. First, PFM-AFM provides label-free imaging of living cells in fully native conditions that allows to obtain otherwise inaccessible information about the effect of cytoskeletal conformations on cell stiffness. In the context of cancer cell mechanics, this information is crucial for understanding the underlying causes of the disparate mechanical properties between normal and tumorigenic cells. Second, for the case of the three epithelial breast cells with different degree of malignancy considered here, the combined interpretation of PFM-AFM and immunofluorescence confocal microscopy imaging results substantiates that F-actin stress fibers provide a dominant contribution to cell stiffness in healthy cells as probed by AFM indentations, while the elasticity of cancer cells appears mostly unaffected by these structures. Finally, from the comparison of cell stiffness determined by either low loading rate indentations or high frequency PFM-AFM experiments, it follows that whereas at low frequencies each cell line provides slightly differentiated estimations of apparent Young's modulus, at high frequencies only healthy cells are distinguishable. Healthy cells result indeed in an enhanced ratio of stiffness to any of the tumor cells, which allows a more reliable discrimination of healthy against tumorigenic ones. However, cell stiffness as measured in the presented high frequency PFM-AFM experiments does not represent an effective biomechanical hallmark of the degree of malignancy or metastatic potential, as the two cancerous cell lines result mechanically undistinguishable. 


\section{METHODS}

Cell Culture and Sample Preparation. For comparative purposes MCF-7 and MDAMB-231 cell lines were used as representatives of epithelial breast cancer cell lines, and MCF-10A as their healthy counterparts (ECACC, Salisbury, Wiltshire, UK). MCF-7 and MDA-MB-231 cells were grown in Dulbecco's modified Eagle's medium (DMEM, Gibco, Life Technologies Corporation, Rockville, MD, USA) supplemented with 10\% FBS, 500 $\mathrm{UI} / \mathrm{ml}$ penicillin and $0.1 \mathrm{mg} / \mathrm{ml}$ streptomycin. MCF-10A cells were maintained in HAM/F12 medium (DMEM/F12, Gibco) supplemented with 5\% horse serum, $20 \mathrm{ng} / \mathrm{ml}$ epidermal growth factor, $0.5 \mu \mathrm{g} / \mathrm{ml}$ hydrocortisone, $100 \mathrm{ng} / \mathrm{ml}$ cholera toxin, $10 \mu \mathrm{g} / \mathrm{ml}$ insulin, and 500 $\mathrm{UI} / \mathrm{ml}$ penicillin and $0.1 \mathrm{mg} / \mathrm{ml}$ streptomycin. Cells were maintained at $37^{\circ} \mathrm{C}$ in $5 \% \mathrm{CO}_{2}$ in a humidified incubator.

Atomic Force Microscopy. All data were acquired in liquid environment using a Bioscope Catalyst AFM (Bruker, Santa Barbara, CA) mounted on a Leica SPE inverted microscope (Leica, Wetzlar, Germany). One day prior to the experiments, cells were seeded at a density of $2 \times 10^{5}$ cells ml $^{-1}$ onto $35 \mathrm{~mm}$ cell culture plates (Corning Inc, Corning, NY, US). To ensure that the cells were in physiological conditions and adherent during the imaging, all the measurements were performed using a home-made heater that maintained the cell cultures at $37^{\circ} \mathrm{C}$. In order to obtain quantitative data, cantilever spring constants were experimentally determined by the thermal tuning method. PFM-AFM was performed by using the Peak-Force Tapping ${ }^{T M}$ feature of the microscope and silicon nitride cantilevers (MLCT-E, Bruker ${ }^{\mathrm{TM}}$ ) with a nominal spring constant of $0.1 \mathrm{~N} / \mathrm{m}$ and a pyramidal tip with a nominal half angle of $18^{\circ}$ and tip radius of $20 \mathrm{~nm}$ in order to provide high lateral resolution. We used a setpoint peak-force in the range of $0.7-1 \mathrm{nN}$. The z-piezo position was oscillated at $0.25 \mathrm{kHz}$, and the amplitude of the oscillation was set at $2 \mathrm{um}$. The commercial software includes a function that removes in real time the hysteresis effect caused in FD curves by hydrodynamic damping of the liquid media. Before image acquisition, the probe lifts up to a height far from the sample, and from there it performs an approach-retract cycle down to a user-defined limit height before tip-sample contact. From this cycle, the effect on the FD curves caused by the liquid media is quantitatively estimated. Then, while scanning for image acquisition, this effect is subtracted in real time from every measured FD curve. We find that the use of relatively stiff cantilevers $(0.1-0.5 \mathrm{~N} / \mathrm{m})$ helps the proper operation of this function. In some cases, a small amount of residual hysteresis remains. However, we find a negligible 
effect of this hydrodynamic damping hysteresis on the estimation of the apparent Young's modulus. The reason is that tip-surface indentation is calculated as the difference between the z-piezo position and the cantilever deflection. Then, the origin of the indentation axis is determined by identifying the contact point as the inflexion point in the curve, and this is done separately for approach and retract curves. When approach and retract force-indentation curves are plotted together with this offset correction, we always observe matching slopes among then, regardless of the presence or not of any hysteresis in the original FD curves. For constant low loading rate indentation experiments we used the standard force-curve acquisition software of the microscope and sQube ${ }^{\circledR}$ colloidal probes (Nanoandmore GMBH) with a borosilicate-glass sphere tip of $10 \mu \mathrm{m}$ diameter and cantilevers with a nominal spring constant of $0.2 \mathrm{~N} / \mathrm{m}$. These spherical indenters have a tip-size that is comparable in order of magnitude to that of the cells, so that they induce global deformations that probe their overall stiffness. Typically 10 cells per line were mechanically characterized by low-loading rate indentations. Force-indentation curves (z-scan size of $2 \mu \mathrm{m}$ ) were recorded at $1 \mathrm{~Hz}$ loading rates with a maximum loading force of $1 \mathrm{nN}$. The apparent Young's modulus of the cells was calculated in this case by fitting the experimental data to a linearized Hertz model equation for spherical indenters:

$$
F=\frac{4}{3} \frac{E}{\left(1-v^{2}\right)} R^{1 / 2} \cdot \delta^{3 / 2}
$$

Immunofluorescence Assays. Cells were seeded at a density of $2 \times 10^{5}$ cells $/ \mathrm{ml}$ onto IbiTreat 35-mm $\mu$-Dish (Ibidi, Martinsried, Germany) and incubated for $24 \mathrm{~h}$. The cell monolayer was washed with PBS, fixed with $4 \%$ (w/v) paraformaldehyde in PBS and permeabilized with $0.1 \%$ Triton X-100 in PBS. For immunostaining, cells were blocked in PBS containing 2\% bovine serum albumin (BSA) and 0.05\% Tween 20 and then incubated with mouse anti-human acetylated $\alpha$-tubulin monoclonal antibody (Sigma-Aldrich, Madrid, Spain) diluted 1:50 (v/v) in PBS containing 1\% BSA. After washing with $0.05 \%$ Tween in PBS, cells were stained with goat anti-mouse Alexa-Fluor 594 secondary antibodies (Molecular Probes, Leiden, Holland) diluted 1:1000 (v/v) in PBS containing 1\% BSA. Cells were also incubated with $0.4 \mu \mathrm{M}$ phalloidin-in PBS to visualize F-actin. After washing with $0.05 \%$ Tween in PBS, cells were examined using a confocal microscope setup separated from the AFM system (Leica SP5, Wetzlar, Germany). 


\section{AUTHOR INFORMATION}

\section{*Corresponding author's contact information:}

E-mail: alvaro.sanpaulo@csic.es

\section{Author Contributions}

The manuscript was written through contributions of all authors. All authors have given approval to the final version of the manuscript. $\ddagger$ These authors contributed equally.

\section{ACKNOWLEDGEMENT}

This work was supported by the ERC Starting Grant "NANOFORCELLS" (ERC-StG2011-278860) and by the project "FORCE-FOR-FUTURE" (CSD2010-00024).

Supporting Information Available: Determination of apparent Young's modulus for each cell line from low loading rate indentations at $1 \mathrm{~Hz}$ with pyramidal sharp tips (Figure $\mathrm{S} 1$ ); Details of the statistics of apparent Young's modulus for each cell line (Figure S2); Additional PFM-AFM images for each cell line (Figures S3, S4, S5); Additional confocal microscopy images for each cell line (Figure S6); Extended description of the used contact mechanics models. 


\section{REFERENCES}

1. Davies, P. F.; Polacek, D. C.; Handen, J. S.; Helmke, B. P.; DePaola, N. A Spatial Approach to Transcriptional Profiling: Mechanotransduction and the Focal Origin of Atherosclerosis. Trends Biotechnol. 1999, 17, 347-351.

2. Ingber, D. E. Mechanobiology and Diseases of Mechanotransduction. Ann. Med. 2003, 35, 564-577.

3. Jaalouk, D. E.; Lammerding, J. Mechanotransduction Gone Awry. Nat. Rev. Mol. Cell Biol. 2009, 10, 63-73.

4. Steward, R. L., Jr.; Rosner, S. R.; Zhou, E. H.; Fredberg, J. J. Illuminating Human Health through Cell Mechanics. Swiss Med. Wkly. 2013, 143.

5. Suresh, S.; Spatz, J.; Mills, J. P.; Micoulet, A.; Dao, M.; Lim, C. T.; Beil, M.; Seufferlein, T. Connections between Single-Cell Biomechanics and Human Disease States: Gastrointestinal Cancer and Malaria. Acta. Biomater. 2005, 1, 1530.

6. Fritsch, A.; Hockel, M.; Kiessling, T.; Nnetu, K. D.; Wetzel, F.; Zink, M.; Kas, J. A. Are Biomechanical Changes Necessary for Tumour Progression? Nat. Phys. 2010, 6, 730-732.

7. Katira, P.; Zaman, M. H.; Bonnecaze, R. T. How Changes in Cell Mechanical Properties Induce Cancerous Behavior. Phys. Rev. Lett. 2012, 108, 028103.

8. Wirtz, D.; Konstantopoulos, K.; Searson, P. C. The Physics of Cancer: The Role of Physical Interactions and Mechanical Forces in Metastasis. Nat. Rev. Cancer 2011, 11, 512-522.

9. Michor, F.; Liphardt, J.; Ferrari, M.; Widom, J. What Does Physics Have to Do with Cancer? Nat. Rev. Cancer 2011, 11, 657-670.

10. Bao, G.; Suresh, S. Cell and Molecular Mechanics of Biological Materials. Nat. Mater. 2003, 2, 715-725.

11. Moeendarbary, E.; Harris, A. R. Cell Mechanics: Principles, Practices, and Prospects. Wiley Interdiscip. Rev.-Syst. Biol. 2014, 6, 371-388.

12. Lim, C. T.; Zhou, E. H.; Li, A.; Vedula, S. R. K.; Fu, H. X., Experimental Techniques for Single Cell and Single Molecule Biomechanics. 2006; Vol. 26, p 1278-1288.

13. Agus, D. B.; Alexander, J. F.; Arap, W.; Ashili, S.; Aslan, J. E.; Austin, R. H.; Backman, V.; Bethel, K. J.; Bonneau, R.; Chen, W. C.; Chen-Tanyolac, C.; Choi, N. C.; Curley, S. A.; Dallas, M.; Damania, D.; Davies, P. C. W.; Decuzzi, P.; Dickinson, L.; Estevez-Salmeron, L.; Estrella, V. et al.; Phys Sci Oncology Ctr, N. A Physical Sciences Network Characterization of Non-Tumorigenic and Metastatic Cells. Scientific Reports 2013, 3.

14. Azeloglu, E. U.; Costa, K. D. Atomic Force Microscopy in Mechanobiology: Measuring Microelastic Heterogeneity of Living Cells. Methods Mol. Biol. 2011, 736, 303-329.

15. Haase, K.; Pelling, A. E. Investigating Cell Mechanics with Atomic Force Microscopy. J. R. Soc. Interface $2015,12$.

16. Lekka, M.; Laidler, P.; Gil, D.; Lekki, J.; Stachura, Z.; Hrynkiewicz, A. Z. Elasticity of Normal and Cancerous Human Bladder Cells Studied by Scanning Force Microscopy. Eur. Biophys. J. Biophy. 1999, 28, 312-316.

17. Lekka, M.; Pogoda, K.; Gostek, J.; Klymenko, O.; Prauzner-Bechcicki, S.; Wiltowska-Zuber, J.; Jaczewska, J.; Lekki, J.; Stachura, Z. Cancer Cell Recognition - Mechanical Phenotype. Micron 2012, 43, 1259-1266.

18. Dokukin, M. E.; Sokolov, I. On the Measurements of Rigidity Modulus of Soft Materials in Nanoindentation Experiments at Small Depth. Macromolecules 2012, 45, 4277-4288.

19. Pogoda, K.; Jaczewska, J.; Wiltowska-Zuber, J.; Klymenko, O.; Zuber, K.; Fornal, M.; Lekka, M. Depth-Sensing Analysis of Cytoskeleton Organization Based on Afm Data. Eur. Biophys. J. Biophy. 2012, 41, 79-87.

20. Li, Q. S.; Lee, G. Y. H.; Ong, C. N.; Lim, C. T. Afm Indentation Study of Breast Cancer Cells. Biochem. Biophys. Res. Commun. 2008, 374, 609-613.

21. Gavara, N.; Chadwick, R. S. Determination of the Elastic Moduli of Thin Samples and Adherent Cells Using Conical Atomic Force Microscope Tips. Nat. Nanotech. 2012, 7, 733-736.

22. Guz, N.; Dokukin, M.; Kalaparthi, V.; Sokolov, I. If Cell Mechanics Can Be Described by Elastic Modulus: Study of Different Models and Probes Used in Indentation Experiments. Biophys. J. 2014, 107, 564-575. 
23. Lekka, M.; Gil, D.; Pogoda, K.; Dulinska-Litewka, J.; Jach, R.; Gostek, J.; Klymenko, O.; Prauzner-Bechcicki, S.; Stachura, Z.; Wiltowska-Zuber, J.; Okon, K.; Laidler, P. Cancer Cell Detection in Tissue Sections Using Afm. Arch. Biochem. Biophys. 2012, 518, 151-156.

24. Prabhune, M.; Belge, G.; Dotzauer, A.; Bullerdiek, J.; Radmacher, M. Comparison of Mechanical Properties of Normal and Malignant Thyroid Cells. Micron 2012, 43, 1267-1272.

25. Rebelo, L. M.; de Sousa, J. S.; Mendes Filho, J.; Radmacher, M. Comparison of the Viscoelastic Properties of Cells from Different Kidney Cancer Phenotypes Measured with Atomic Force Microscopy. Nanotechnology 2013, 24.

26. Ketene, A. N.; Schmelz, E. M.; Roberts, P. C.; Agah, M. The Effects of Cancer Progression on the Viscoelasticity of Ovarian Cell Cytoskeleton Structures. Nanomed.-Nanotechnol. Biol. Med. 2012, 8, 93-102.

27. Weder, G.; Hendriks-Balk, M. C.; Smajda, R.; Rimoldi, D.; Liley, M.; Heinzelmann, H.; Meister, A.; Mariotti, A. Increased Plasticity of the Stiffness of Melanoma Cells Correlates with Their Acquisition of Metastatic Properties. Nanomed.-Nanotechnol. Biol. Med. 2014, 10, 141-148.

28. Cross, S. E.; Jin, Y.-S.; Rao, J.; Gimzewski, J. K. Nanomechanical Analysis of Cells from Cancer Patients. Nat. Nanotech. 2007, 2, 780-783.

29. Plodinec, M.; Loparic, M.; Monnier, C. A.; Obermann, E. C.; Zanetti-Dallenbach, R.; Oertle, P.; Hyotyla, J. T.; Aebi, U.; Bentires-Alj, M.; Lim, R. Y. H.; Schoenenberger, C. A. The Nanomechanical Signature of Breast Cancer. Nat. Nanotech. 2012, 7, 757-765.

30. Suresh, S. Biomechanics and Biophysics of Cancer Cells. Acta. Biomater. 2007, 3, 413-438.

31. Sawyers, C. L. The Cancer Biomarker Problem. Nature 2008, 452, 548-552.

32. Tojkander, S.; Gateva, G.; Lappalainen, P. Actin Stress Fibers - Assembly, Dynamics and Biological Roles. J. Cell Sci. 2012, 125, 1855-1864.

33. Dufrene, Y. F.; Martinez-Martin, D.; Medalsy, I.; Alsteens, D.; Mueller, D. J. Multiparametric Imaging of Biological Systems by Force-Distance Curve-Based Afm. Nat. Methods 2013, 10, 847-854.

34. Zhang, S.; Aslan, H.; Besenbacher, F.; Dong, M. Quantitative Biomolecular Imaging by Dynamic Nanomechanical Mapping. Chem. Soc. Rev. 2014, 43, 7412-7429.

35. Garcia, R.; Perez, R. Dynamic Atomic Force Microscopy Methods. Surf. Sci. Rep. 2002, 47, 197-301.

36. Cartagena-Rivera, A. X.; Wang, W.-H.; Geahlen, R. L.; Raman, A. Fast, Multi-Frequency, and Quantitative Nanomechanical Mapping of Live Cells Using the Atomic Force Microscope. Scientific Reports 2015, 5.

37. Cartagena, A.; Raman, A. Local Viscoelastic Properties of Live Cells Investigated Using Dynamic and Quasi-Static Atomic Force Microscopy Methods. Biophys. J. 2014, 106, 1033-1043.

38. Raman, A.; Trigueros, S.; Cartagena, A.; Stevenson, A. P. Z.; Susilo, M.; Nauman, E.; Contera, S. A. Mapping Nanomechanical Properties of Live Cells Using Multi-Harmonic Atomic Force Microscopy. Nat. Nanotech. 2011, 6 , 809-814.

39. Garcia, R.; Proksch, R. Nanomechanical Mapping of Soft Matter by Bimodal Force Microscopy. Eur. Polym. J. 2013, 49, 1897-1906.

40. Heu, C.; Berquand, A.; Elie-Caille, C.; Nicod, L. Glyphosate-Induced Stiffening of Hacat Keratinocytes, a Peak Force Tapping Study on Living Cells. J. Struct. Biol. 2012, 178, 1-7.

41. Alsteens, D.; Dupres, V.; Yunus, S.; Latge, J.-P.; Heinisch, J. J.; Dufrene, Y. F. High-Resolution Imaging of Chemical and Biological Sites on Living Cells Using Peak Force Tapping Atomic Force Microscopy. Langmuir 2012, 28, 1673816744.

42. Xia, D.; Zhang, S.; Hjortdal, J. O.; Li, Q.; Thomsen, K.; Chevallier, J.; Besenbacher, F.; Dong, M. Hydrated Human Corneal Stroma Revealed by Quantitative Dynamic Atomic Force Microscopy at Nanoscale. Acs Nano 2014, 8, 6873 6882.

43. Wang, C.; Stanciu, C.; Ehrhardt, C. J.; Yadavalli, V. K. Morphological and Mechanical Imaging of Bacillus Cereus Spore Formation at the Nanoscale. J. Microsc. 2015, 258, 49-58. 
44. Alsteens, D.; Trabelsi, H.; Soumillion, P.; Dufrene, Y. F. Multiparametric Atomic Force Microscopy Imaging of Single Bacteriophages Extruding from Living Bacteria. Nat. Commun. 2013, 4.

45. Osmulski, P.; Mahalingam, D.; Gaczynska, M. E.; Liu, J.; Huang, S.; Horning, A. M.; Wang, C.-M.; Thompson, I. M.; Huang, T. H. M.; Chen, C.-L. Nanomechanical Biomarkers of Single Circulating Tumor Cells for Detection of Castration Resistant Prostate Cancer. Prostate 2014, 74, 1297-1307.

46. Hozic, A.; Rico, F.; Colom, A.; Buzhynskyy, N.; Scheuring, S. Nanomechanical Characterization of the Stiffness of Eye Lens Cells: A Pilot Study. Invest. Ophthalmol. Vis. Sci. 2012, 53, 2151-2156.

47. Pletikapic, G.; Berquand, A.; Radic, T. M.; Svetlicic, V. Quantitative Nanomechanical Mapping of Marine Diatom in Seawater Using Peak Force Tapping Atomic Force Microscopy. J. Phycol 2012, 48, 174-185.

48. Picas, L.; Rico, F.; Deforet, M.; Scheuring, S. Structural and Mechanical Heterogeneity of the Erythrocyte Membrane Reveals Hallmarks of Membrane Stability. Acs Nano 2013, 7, 1054-1063.

49. Eghiaian, F.; Rigato, A.; Scheuring, S. Structural, Mechanical, and Dynamical Variability of the Actin Cortex in Living Cells. Biophys. J. 2015, 108, 1330-1340.

50. Sakai, M. Time-Dependent Viscoelastic Relation between Load and Penetration for an Axisymmetric Indenter. Philos. Mag. A-Phys. Condens. Matter Struct. Defect Mech. Prop. 2002, 82, 1841-1849.

51. Xu, C.; Wang, Y.; Jiang, N.; Yang, H.; Lin, J.; Xie, S. Elasticity Measurement of Breast Cancer Cells by Atomic Force Microscopy. Proc. SPIE 9230 2014, 92300Y.

52. Rotsch, C.; Radmacher, M. Drug-Induced Changes of Cytoskeletal Structure and Mechanics in Fibroblasts: An Atomic Force Microscopy Study. Biophys. J. 2000, 78, 520-535.

53. Tharmann, R.; Claessens, M. M. A. E.; Bausch, A. R. Viscoelasticity of Isotropically Cross-Linked Actin Networks. Phys. Rev. Lett. 2007, 98, 088103.

54. Koenderink, G. H.; Dogic, Z.; Nakamura, F.; Bendix, P. M.; MacKintosh, F. C.; Hartwig, J. H.; Stossel, T. P.; Weitz, D. A. An Active Biopolymer Network Controlled by Molecular Motors. Proc. Natl. Acad. Sci. U. S. A. 2009, 106, $15192-$ 15197.

55. Teng, L. A Mechanics Model of Microtubule Buckling in Living Cells. J. Biomech. 2008, 41, 1722-1729.

56. Lin, Y.-C.; Koenderink, G. H.; MacKintosh, F. C.; Weitz, D. A. Control of Non-Linear Elasticity in F-Actin Networks with Microtubules. Soft Matter 2011, 7, 902-906.

57. Salbreux, G.; Charras, G.; Paluch, E. Actin Cortex Mechanics and Cellular Morphogenesis. Trends Cell Biol. 2012, 22, 536-545.

58. Rother, J.; Noeding, H.; Mey, I.; Janshoff, A. Atomic Force Microscopy-Based Microrheology Reveals Significant Differences in the Viscoelastic Response between Malign and Benign Cell Lines. Open Biol. 2014, 4. 